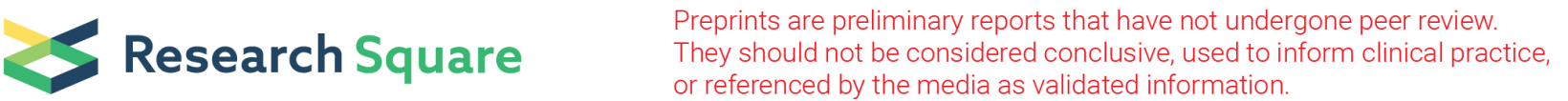 \\ Fast 6D Object Pose Estimation of Shell Parts for Robotic Assembly
}

\section{Haopeng Hu}

Harbin Institute of Technology Shenzhen https://orcid.org/0000-0002-4069-6431

\section{Weikun Gu}

School of Mechanical Engineering and Automation, Harbin Institute of Technology Shenzhen, HIT Campus, University Town of Shenzhen, Xili, Nanshan, Shenzhen, China.

\section{Xiansheng Yang}

School of Mechanical Engineering and Automation, Harbin Institute of Technology Shenzhen, HIT Campus, University Town of Shenzhen, Xili, Nanshan, Shenzhen, China.

\section{Nan Zhang}

School of Mechanical Engineering and Automation, Harbin Institute of Technology Shenzhen, HIT Campus, University Town of Shenzhen, Xili, Nanshan, Shenzhen, China.

\section{Yunjiang Lou ( $\square$ louyj@hit.edu.cn )}

School of Mechanical Engineering and Automation, Harbin Institute of Technology Shenzhen, HIT Campus, University Town of Shenzhen, Xili, Nanshan, Shenzhen, China.

\section{Research Article}

Keywords: 6D pose estimation, Robotic assembly, Point clouds, ICP

Posted Date: March 15th, 2021

DOl: https://doi.org/10.21203/rs.3.rs-285435/v1

License: (c) (i) This work is licensed under a Creative Commons Attribution 4.0 International License. Read Full License

Version of Record: A version of this preprint was published at The International Journal of Advanced Manufacturing Technology on September 16th, 2021. See the published version at https://doi.org/10.1007/s00170-021-07960-0. 


\section{Abstract}

Shell parts which have similar and close inner and outer surfaces are common in industrial manufacturing applications. In view of the $6 \mathrm{D}$ pose error compensation of parts in high-precision robotic assembly tasks, this work proposes a fast $6 \mathrm{D}$ pose estimation approach tailored for shell parts. With a binocular structured light camera, the proposed approach consists of two phases, namely initial pose estimation phase and local pose estimation phase. In the former one, an initial pose correction and translation offset methods serve to solve the local optimal estimation problem of the iterative closest point (ICP) algorithm. This problem is caused by the poorly assigned initial pose and the similar inner and outer surfaces of shell parts. In the latter one, the voxel sampling and the weighted point-to-plane ICP algorithms are applied to boost the efficiency of the pose estimation approach. With two typical shell parts, a simulation and an experiment of pose estimation are conducted to verify the effectiveness of the proposed approach. Experiment results prove that the accuracy of the pose estimation approach is $0: 27 \mathrm{~mm} / 0: 38^{\circ}$, and the runtime is $680 \mathrm{~ms}$.

\section{Full Text}

This preprint is available for download as a PDF.

\section{Figures}

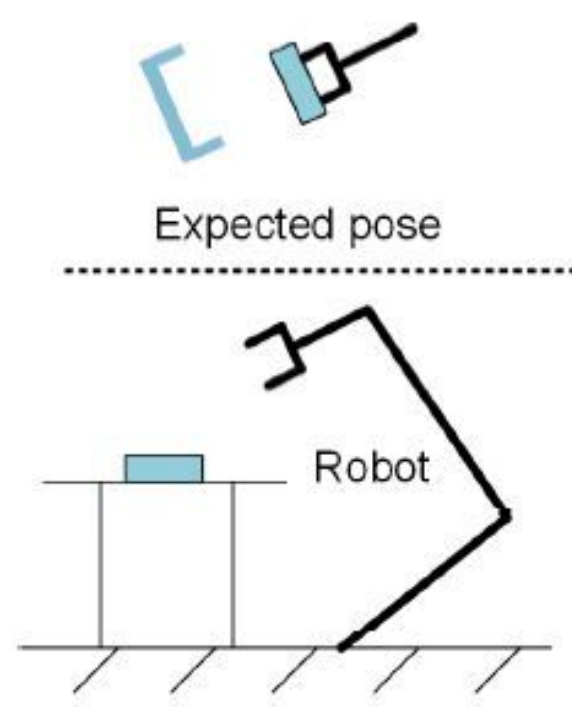

(a) Initial state

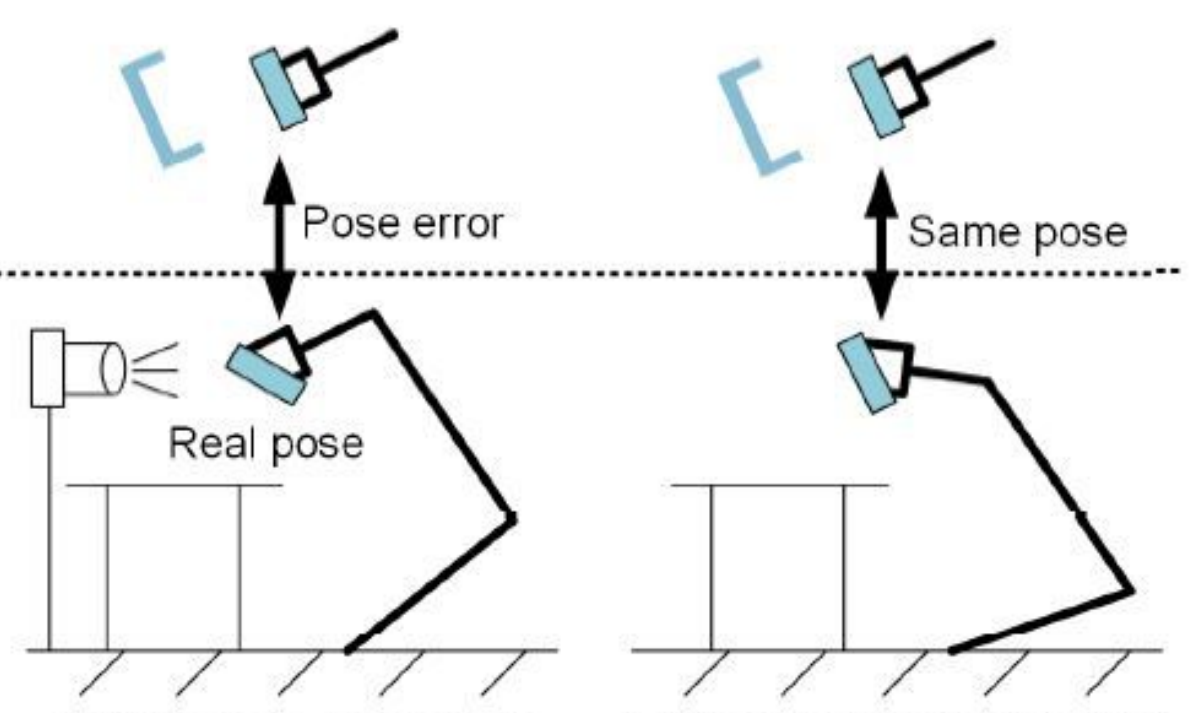

(b) Real pose acquisition (c) Pose error compensation

\section{Figure 1}

Pose compensation in robotic assembly tasks 


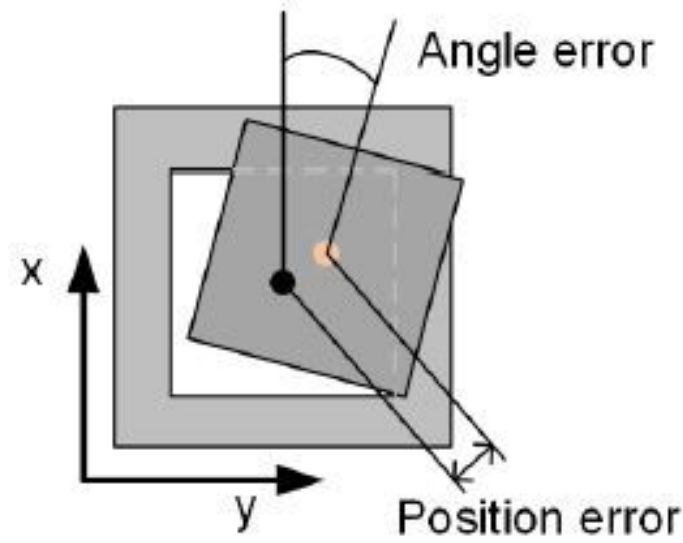

(a) 3D pose error

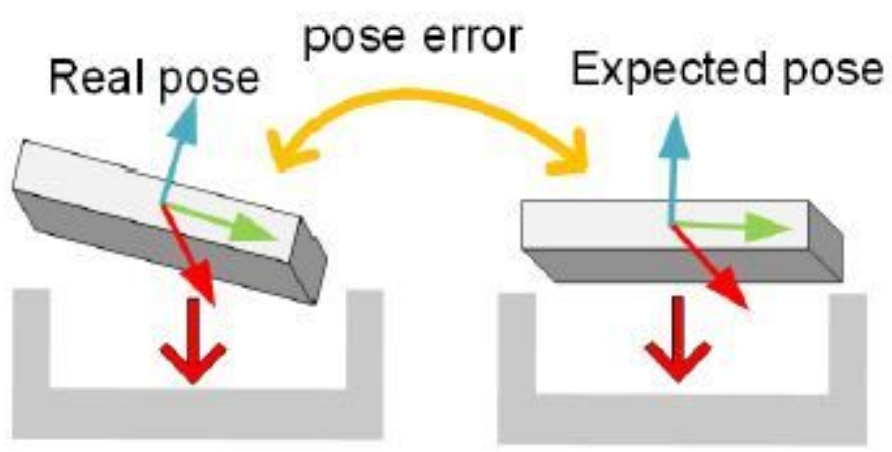

Assembly failure Assembly success

(b) 6D pose error

Figure 2

Pose errors in assembly tasks.
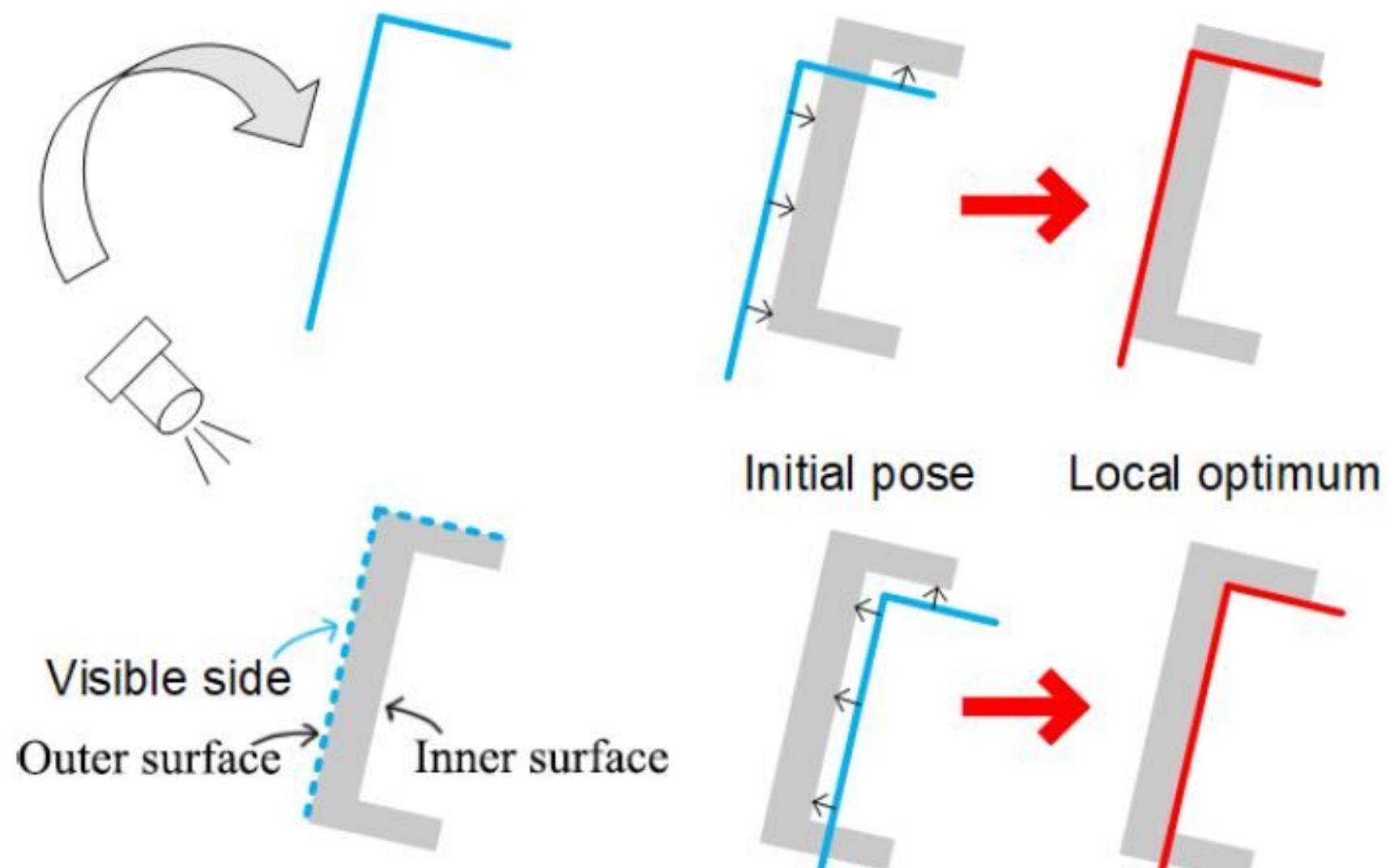

Figure 3

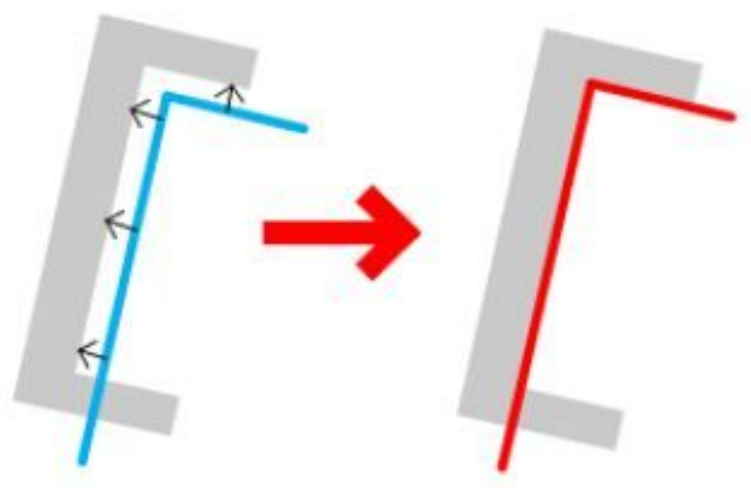


Local optimal estimation problem: Given the shell parts, the 3D vision sensor can only capture the point cloud of its visible side. Owing to the poorly assigned initial poses and the similarity of inner and outer surfaces of shell parts, improper corresponding relationships of point pairs will be identified by ICP, which results in local optimal pose estimation.

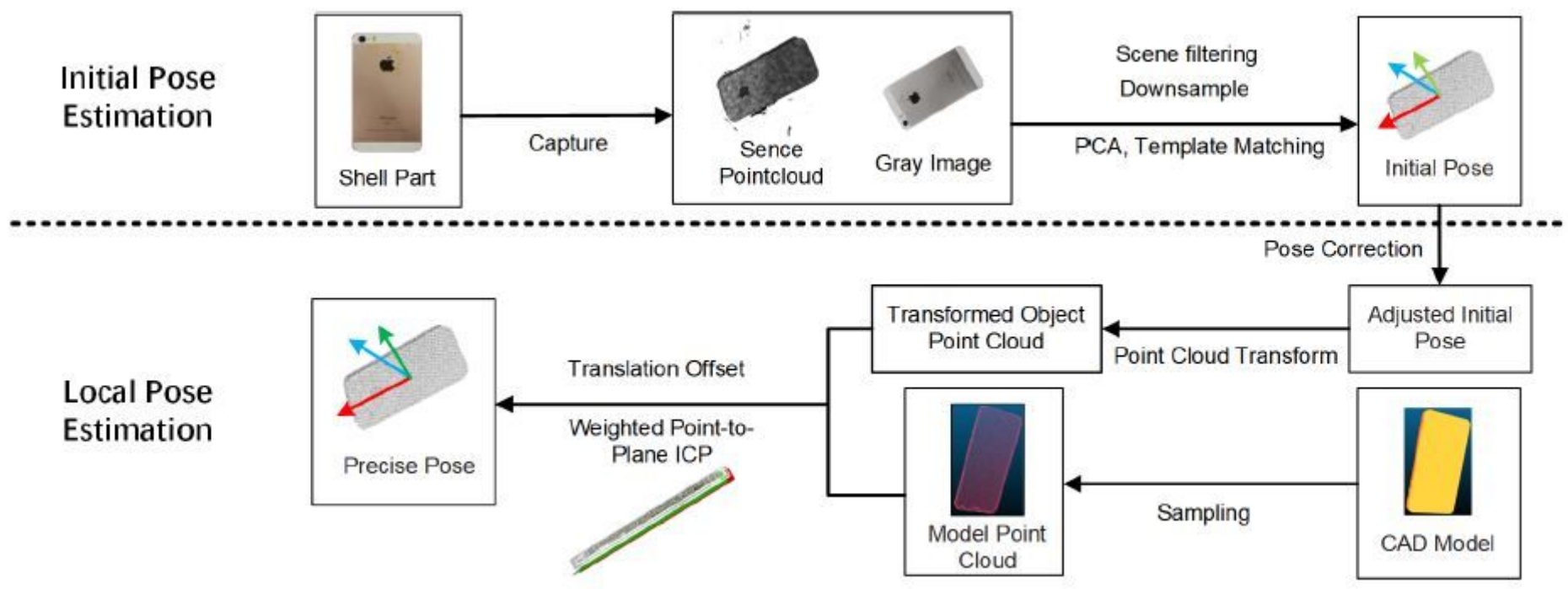

Figure 4

Work flow of the proposed fast $6 \mathrm{D}$ pose estimation approach for shell parts
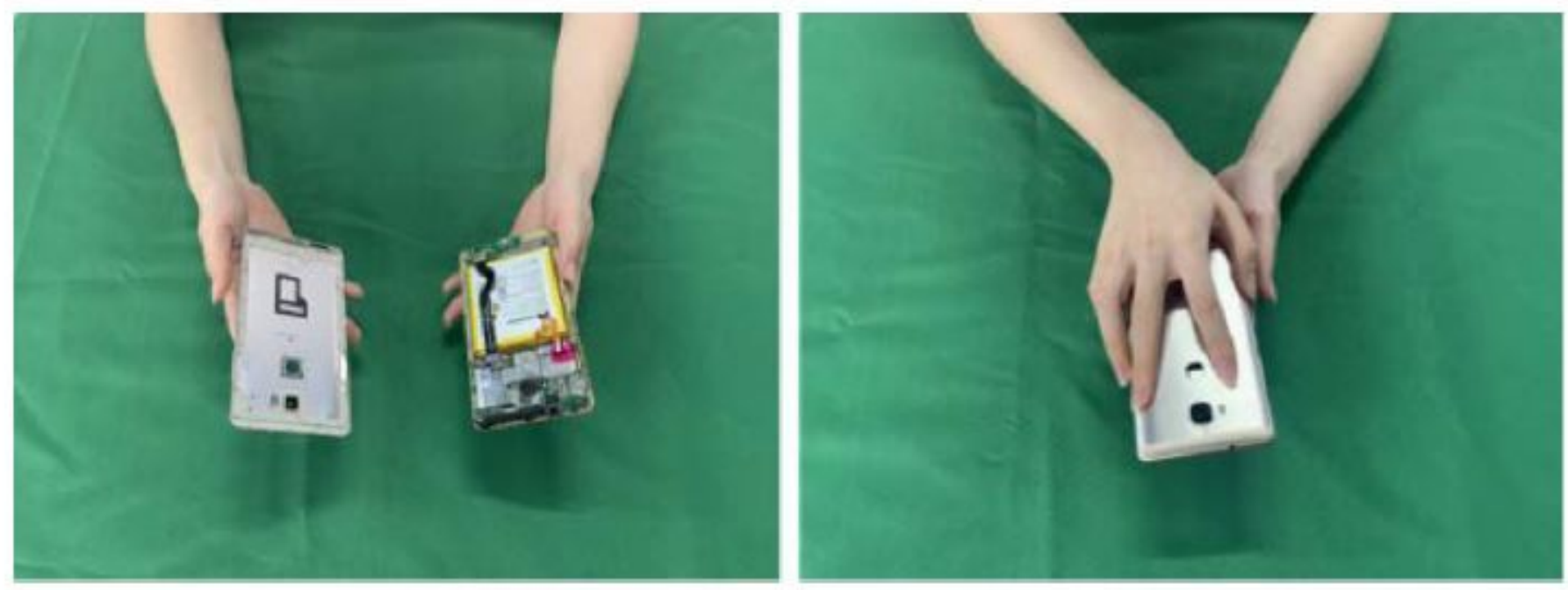

\section{Figure 5}

The manual assembly process of a cellphone back cover. 


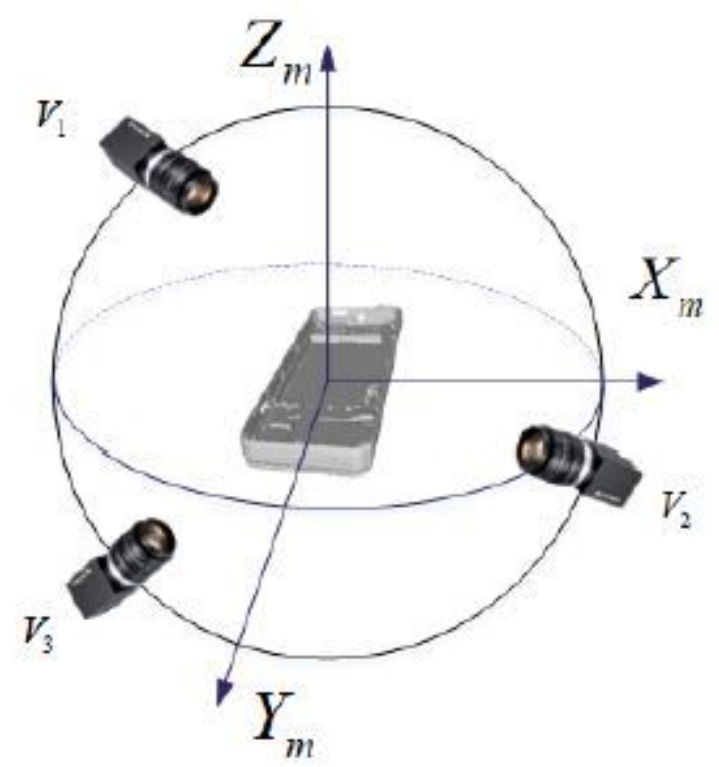

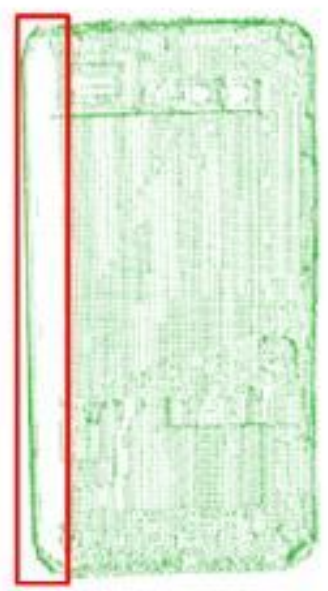

$V_{1}$

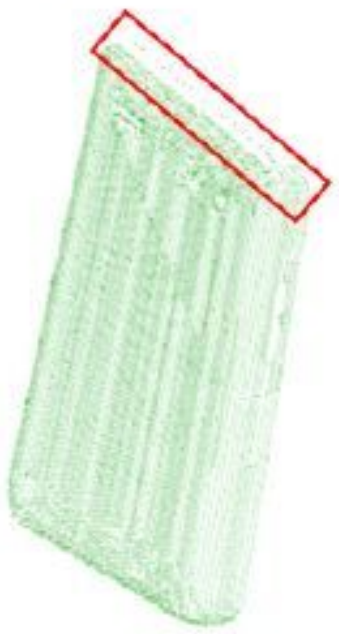

$V_{2}$

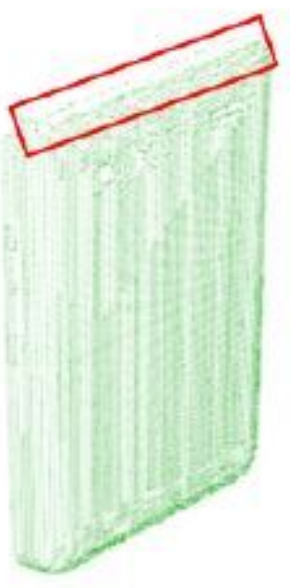

$V_{3}$

Figure 6

Data missing caused by self-occlusion problem: Three viewpoints and the corresponding visible PCD. 


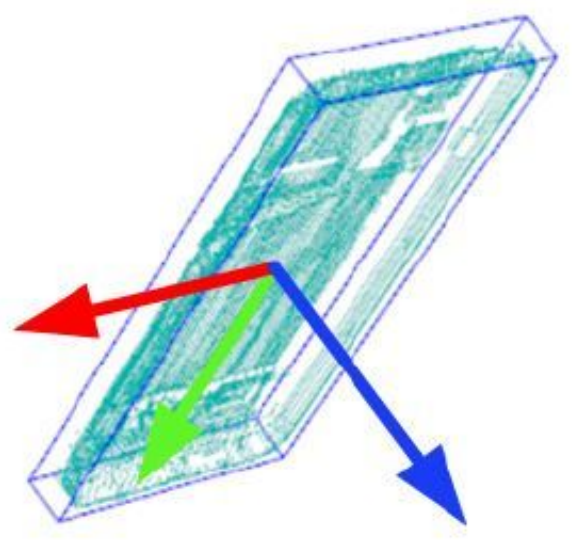

\section{Original Point Cloud}

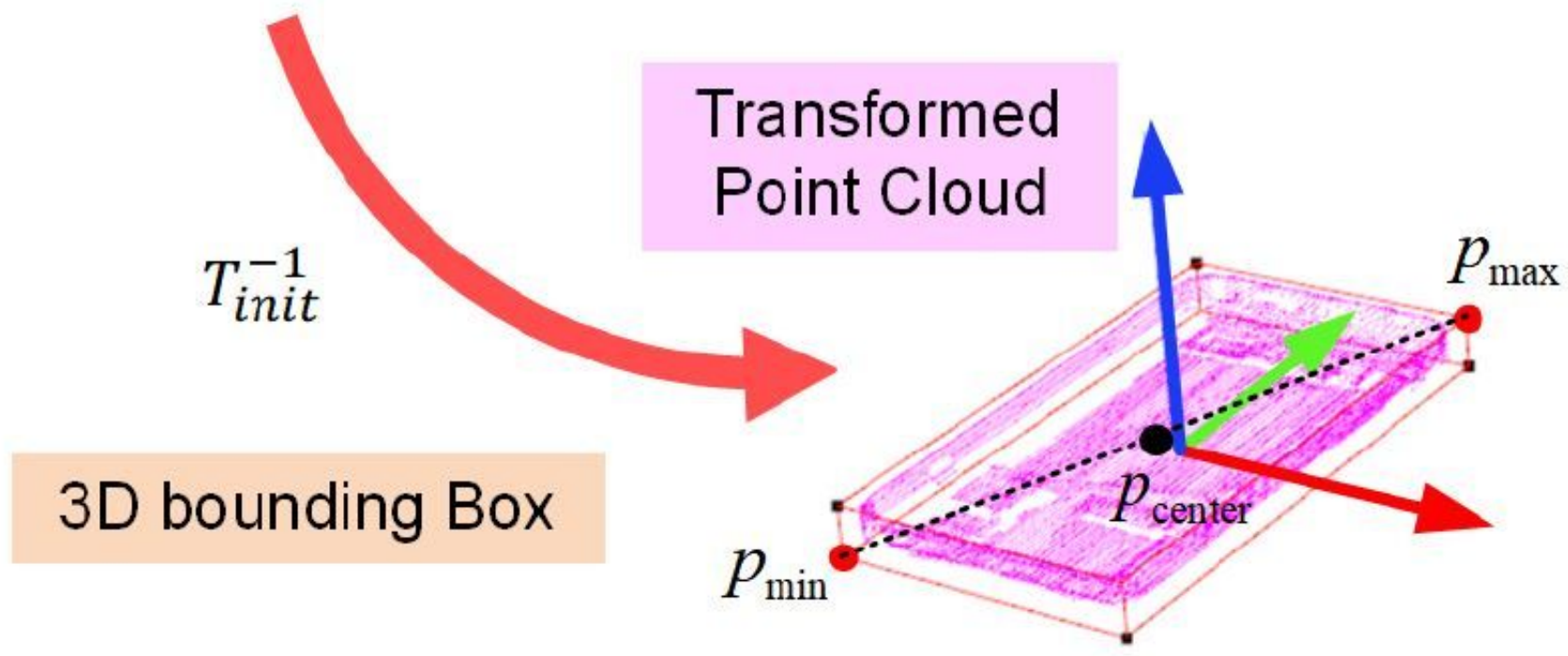

Figure 7

The pose correction process. 

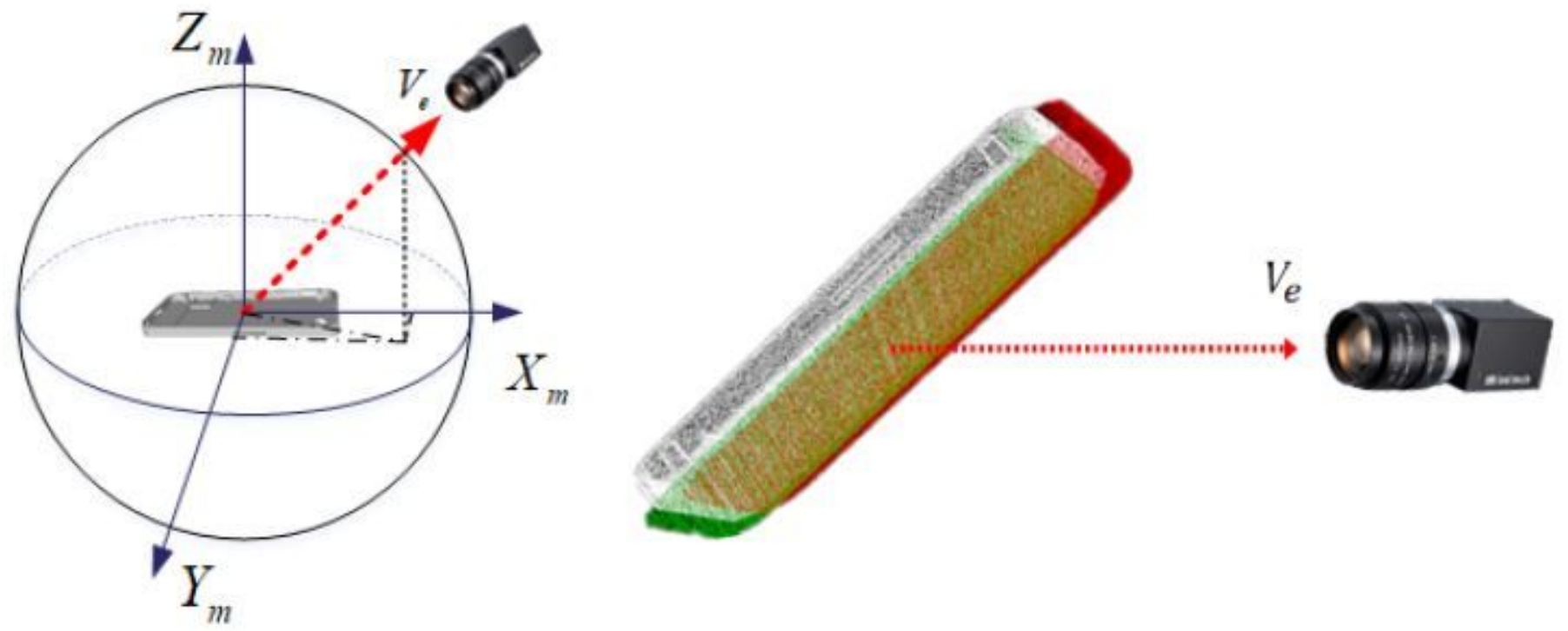

Figure 8

The viewpoint direction (left) and the translation offset (right).

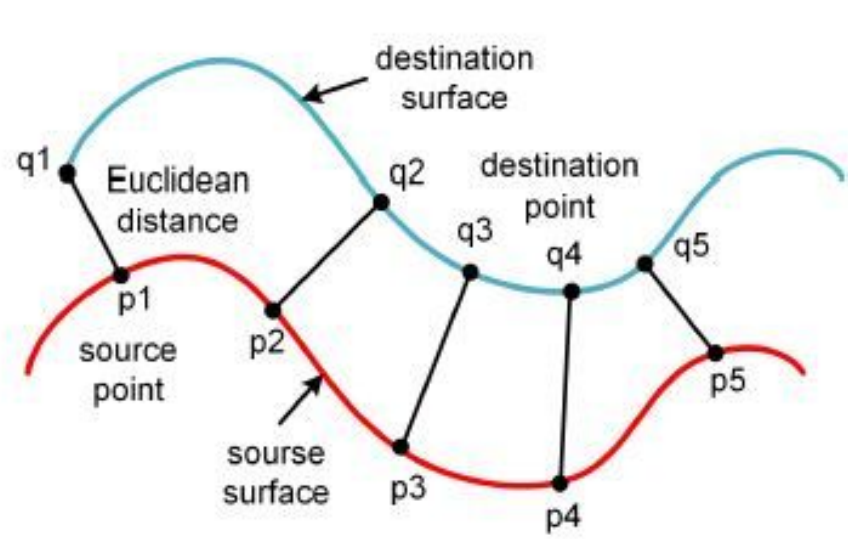

(a) Point-to-point translation

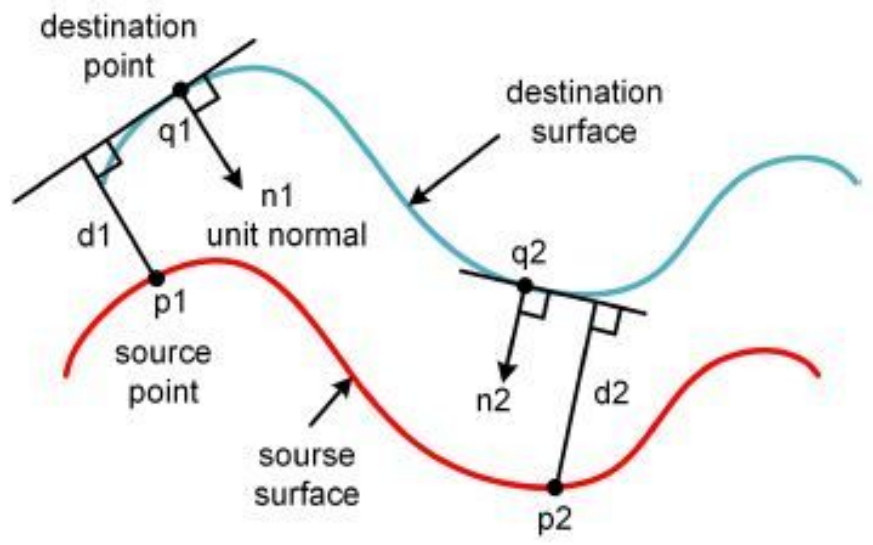

(b) Point-to-plane translation

\section{Figure 9}

Two translation metrics of the corresponding points 

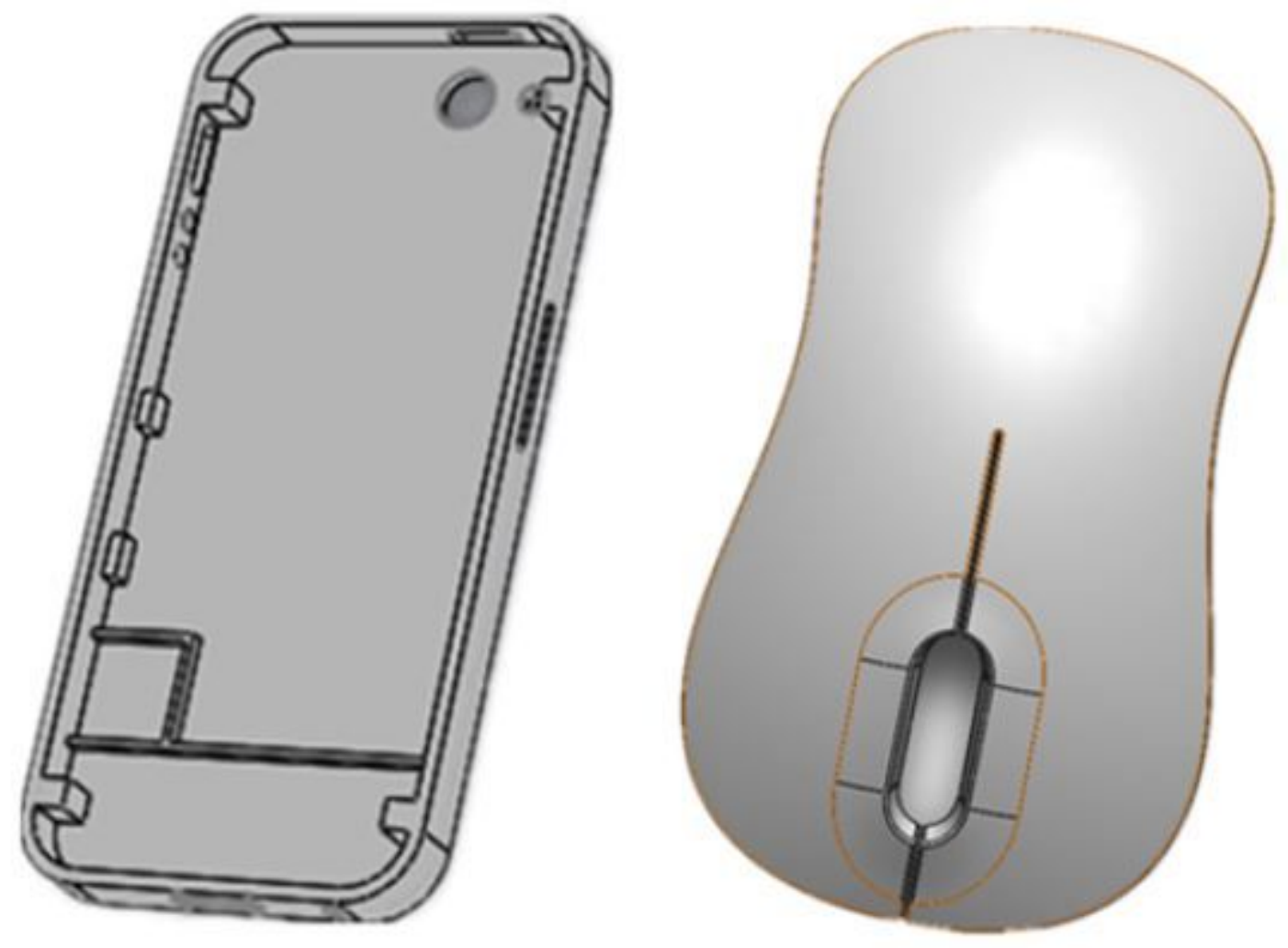

Figure 10

Simulation setup: The CAD models used in the simulation and the elevation rotation of the viewpoints. 


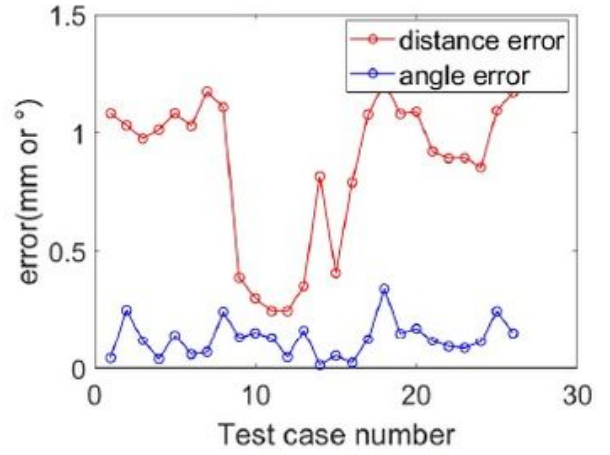

(a) Point-to-point ICP for part A

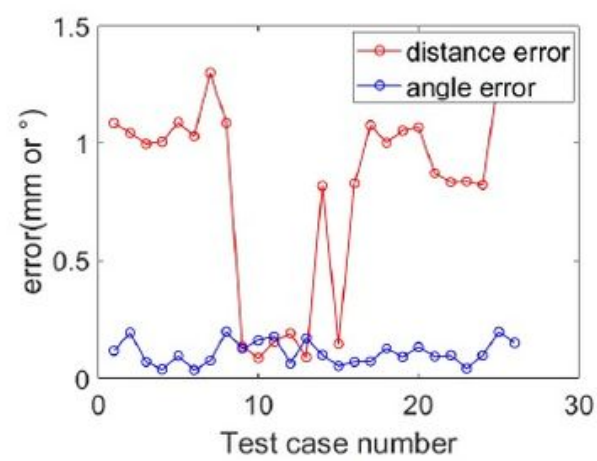

(c) Point-to-plane ICP for part A

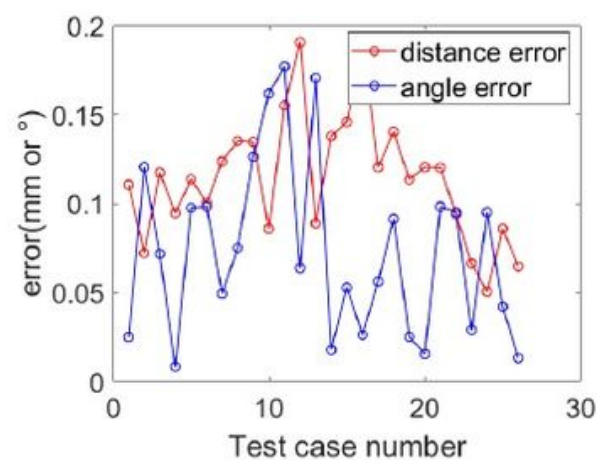

(e) Our approach for part A

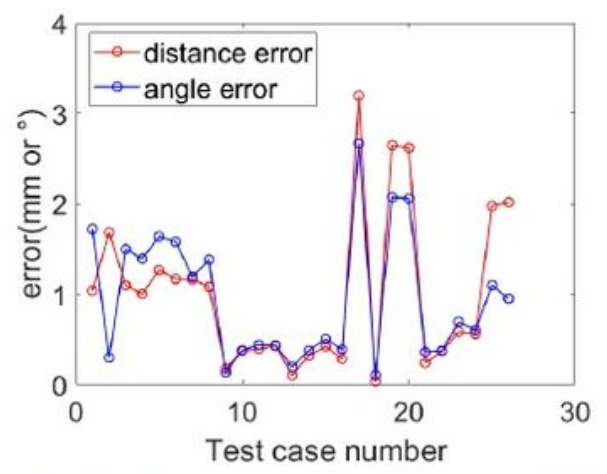

(b) Point-to-point ICP for part B

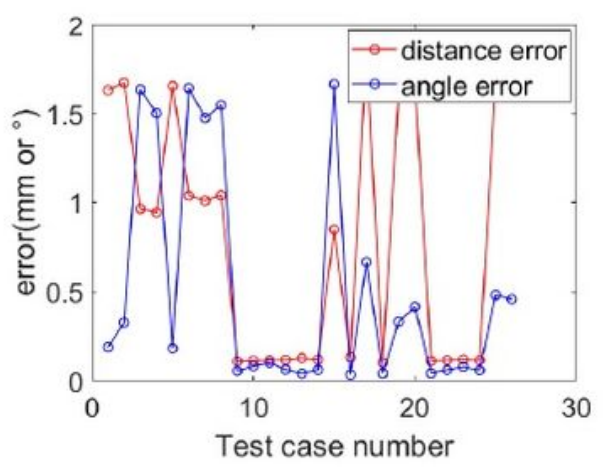

(d) Point-to-plane ICP for part B

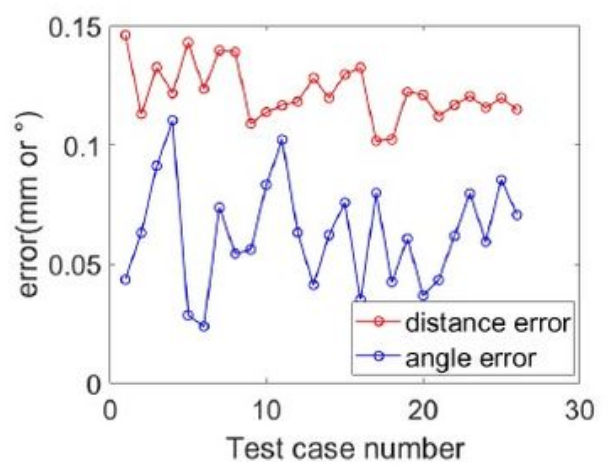

(f) Our approach for part B

\section{Figure 11}

Pose errors of three approaches in each test. 


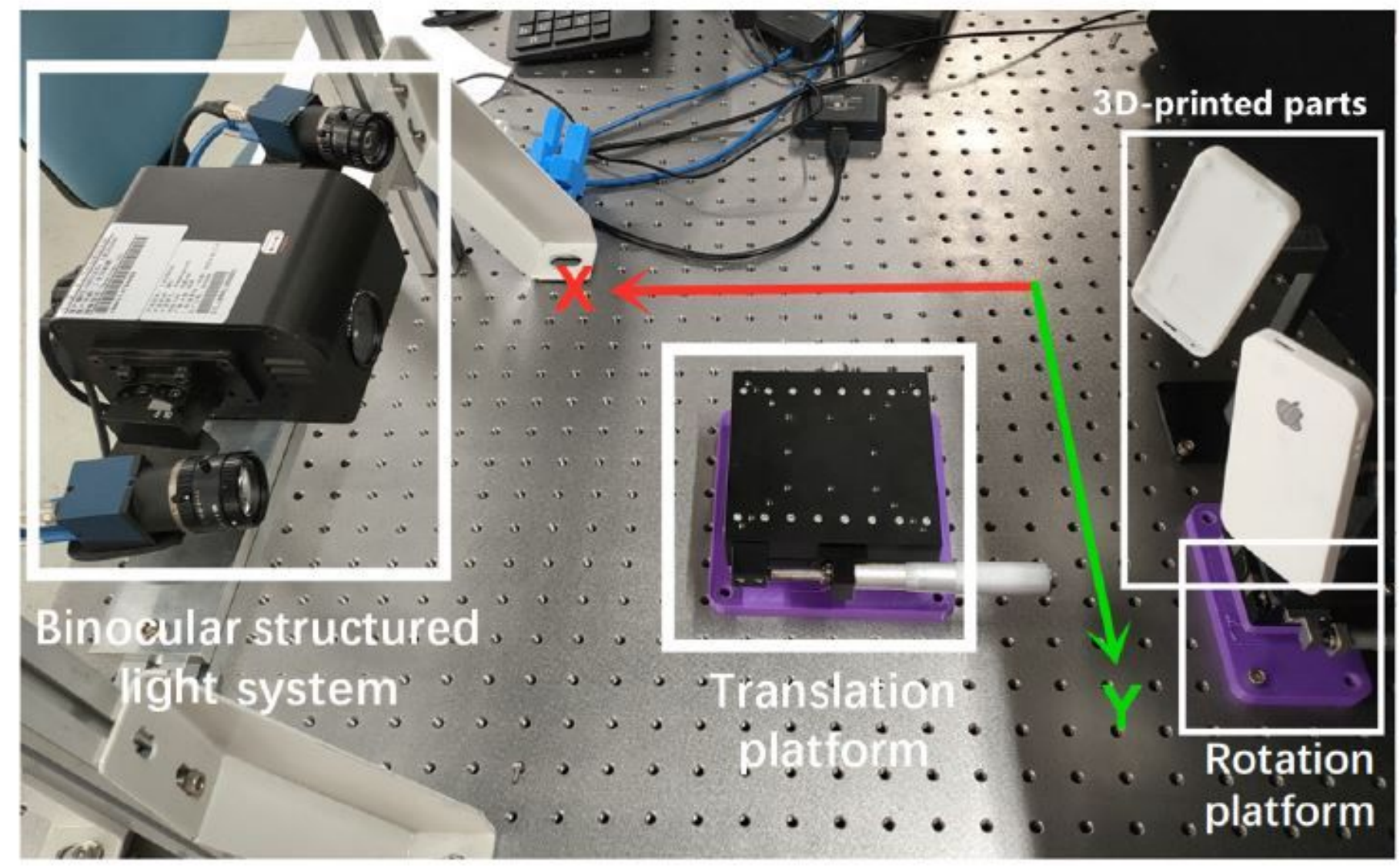

Figure 12

Experimental platform: The $\mathrm{X}$-axis and $\mathrm{Y}$-axis indicate the movement direction of the rotation and translation platforms, respectively.

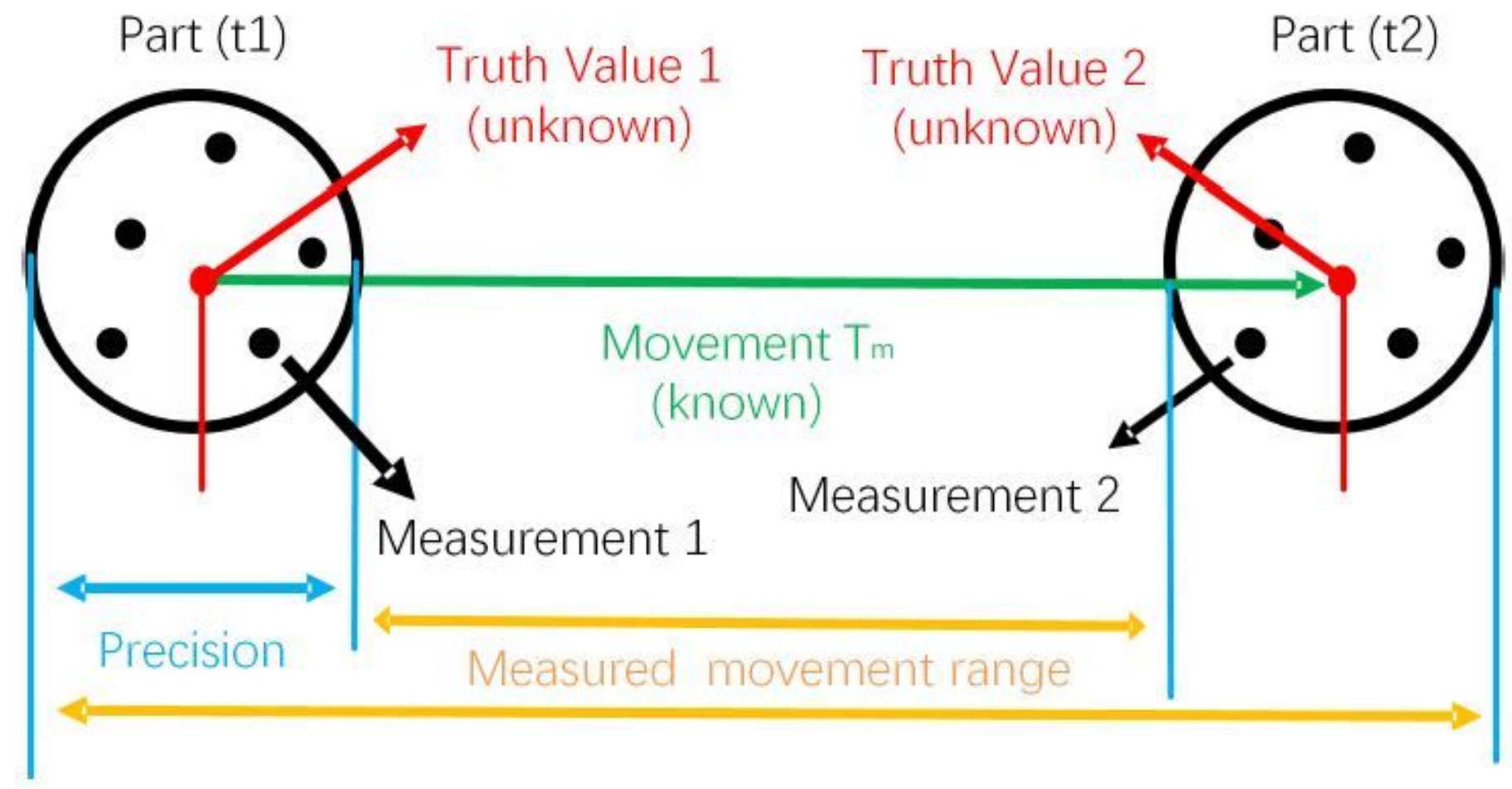


Figure 13

Schematic of precision and accuracy experiments: For pose precision, poses part A and B are estimated multiple times to obtained T1:::Tn. And the variance of these poses is used to represent the precision. For pose accuracy, the parts are captured before and after applying a known movement Tm of the platforms and the error between Tm and the applied movement is used to represent the accuracy.

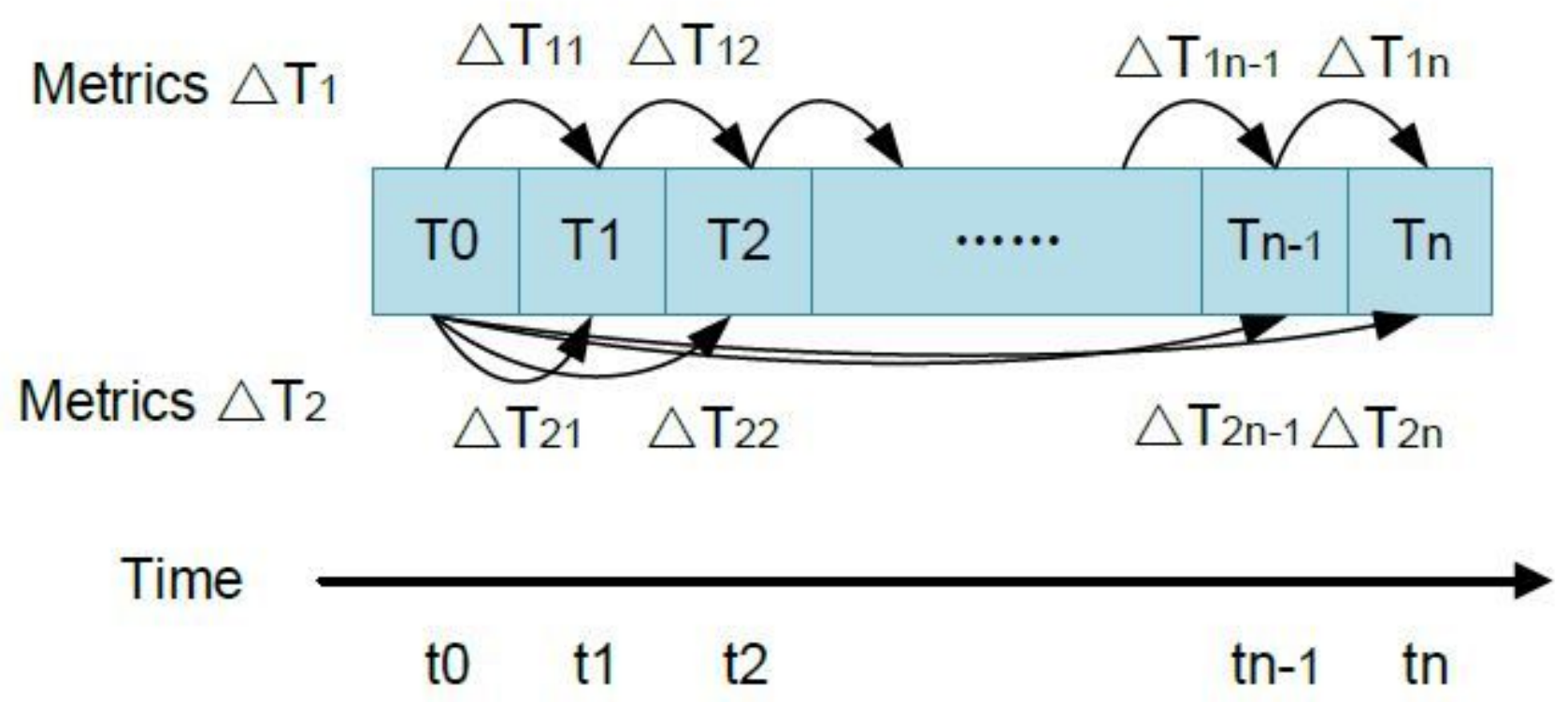

Figure 14

Two metrics for precision and accuracy 


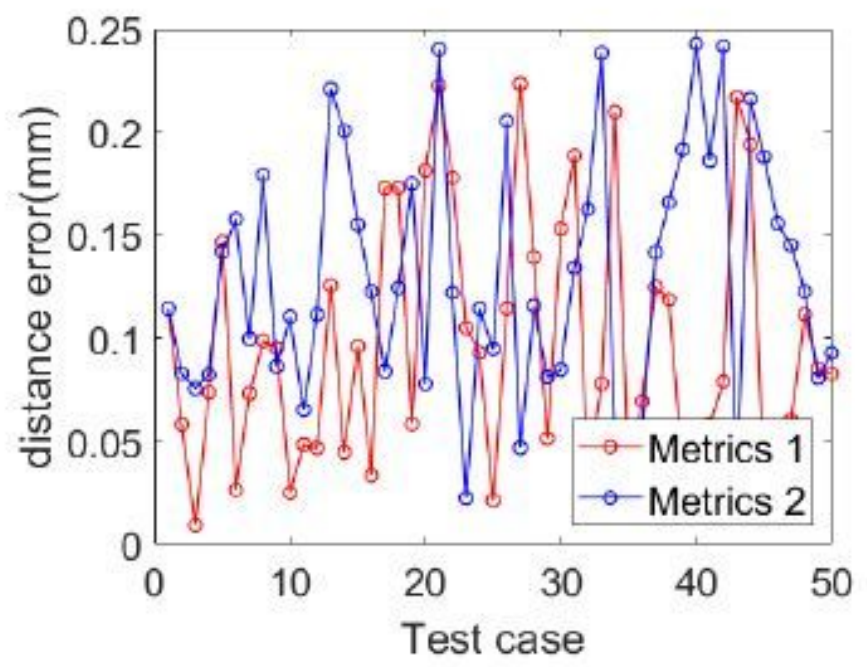

(a) Part A position error

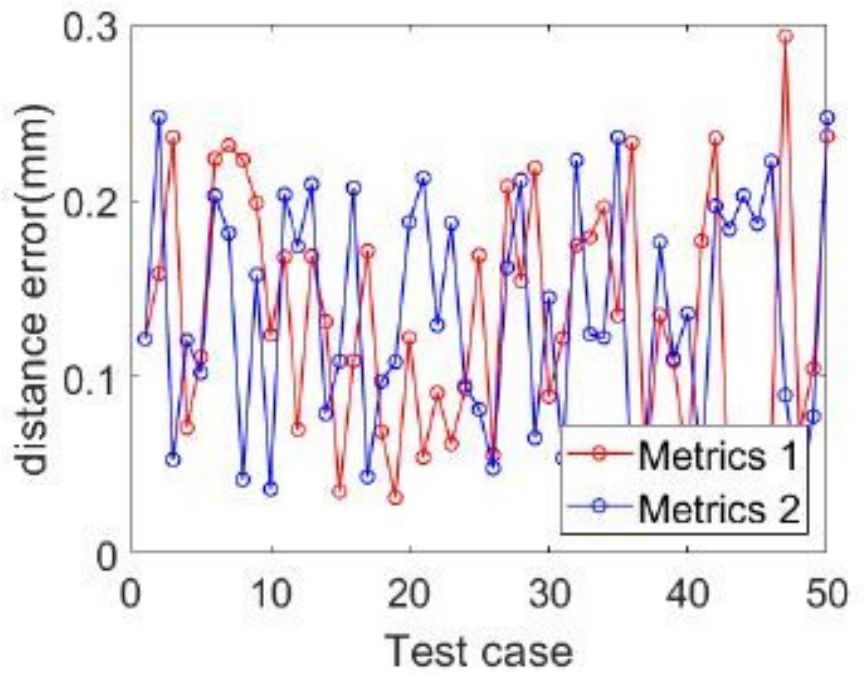

(c) Part B position error

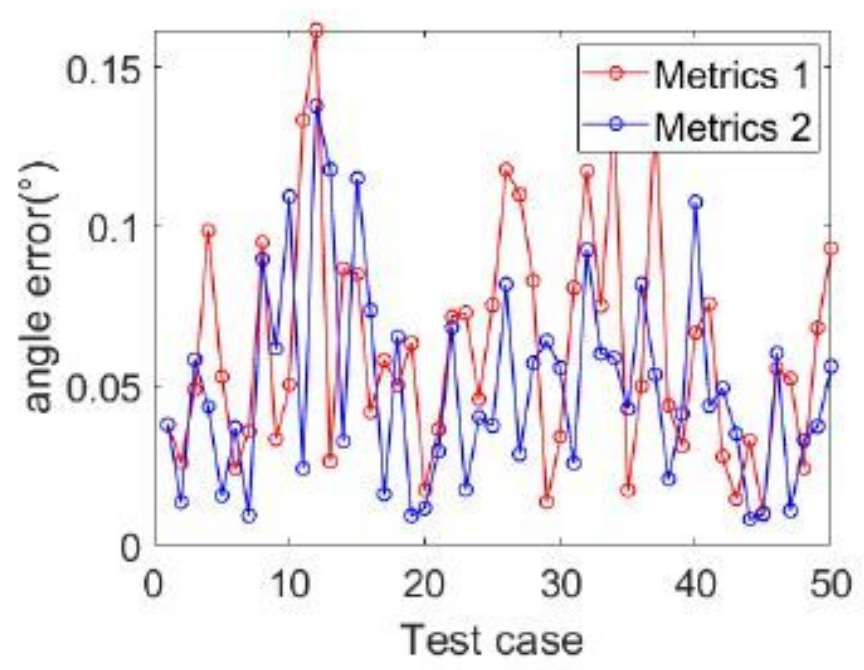

(b) Part A angle error

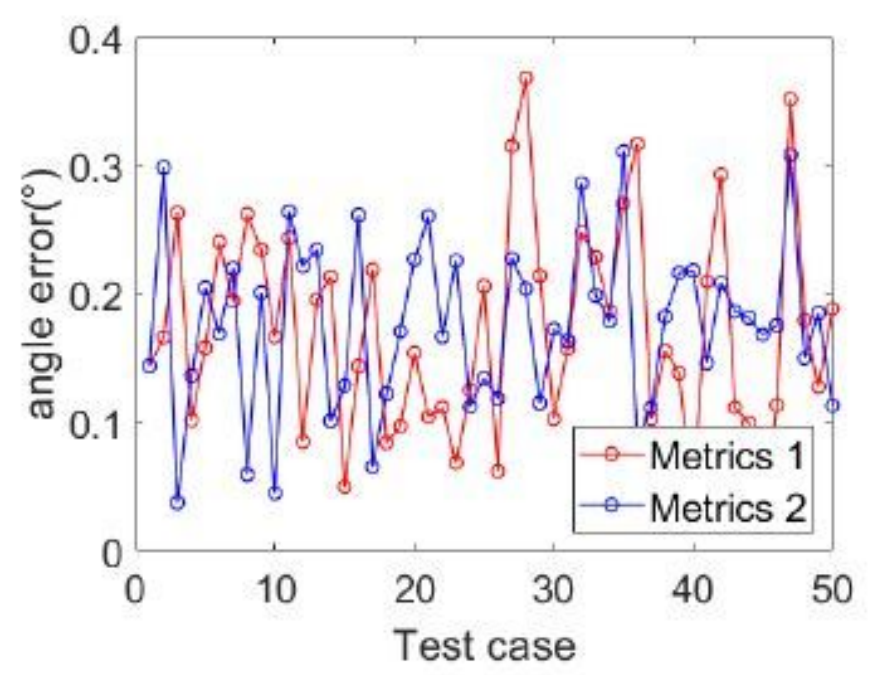

(d) Part B angle error

\section{Figure 15}

Position and angle errors of part $A$ and $B:(a)$ and $(b)$ indicate the position and angle error (precisions) of part A, respectively; (c) and (d) indicate those for part B. The red curve represents metric 1 , and the blue one represents metric 2 . 


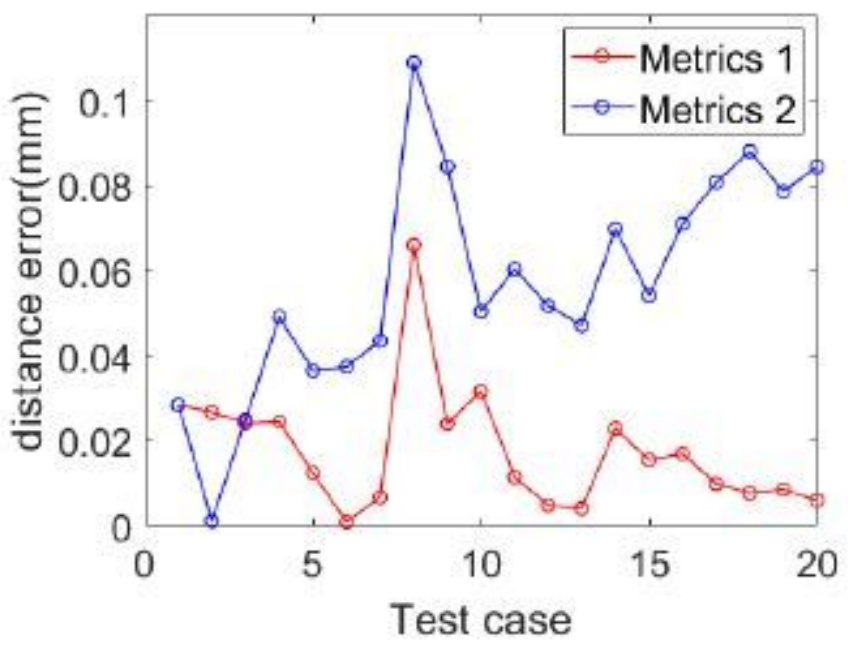

(a) Translation along $\mathrm{X}$-axis

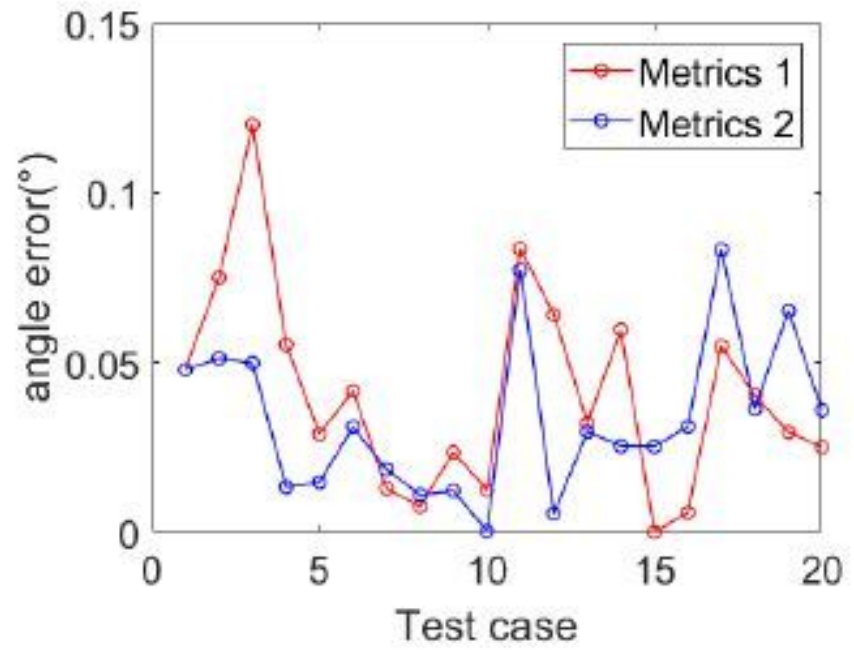

(c) Rotation around $\mathrm{X}$-axis

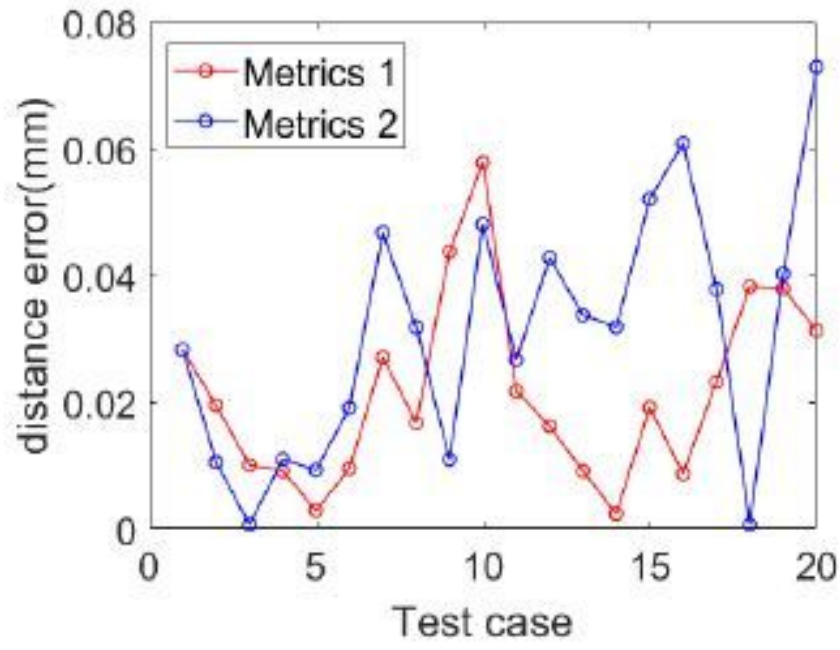

(b) Translation along Y-axis

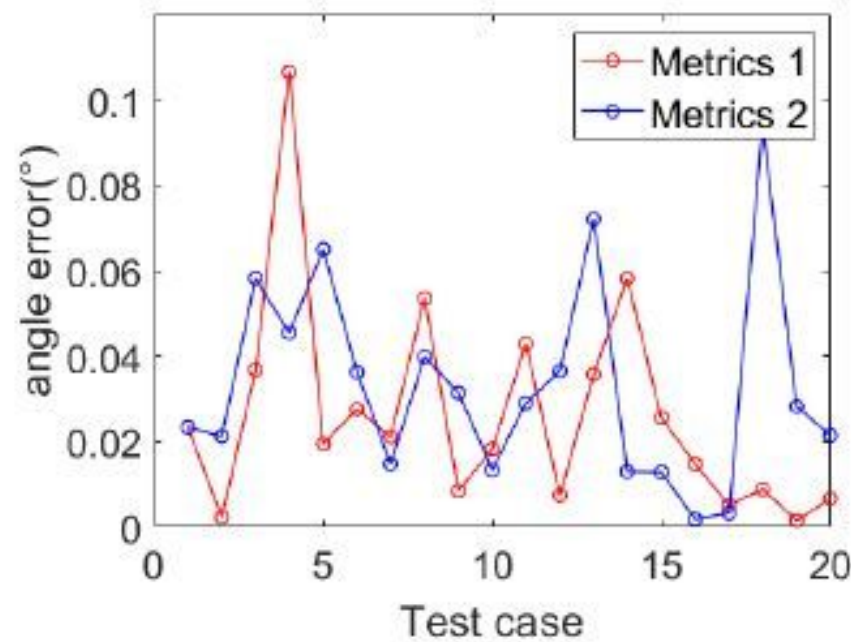

(d) Rotation around Y-axis

Figure 16

Accuracy curves for part A. 

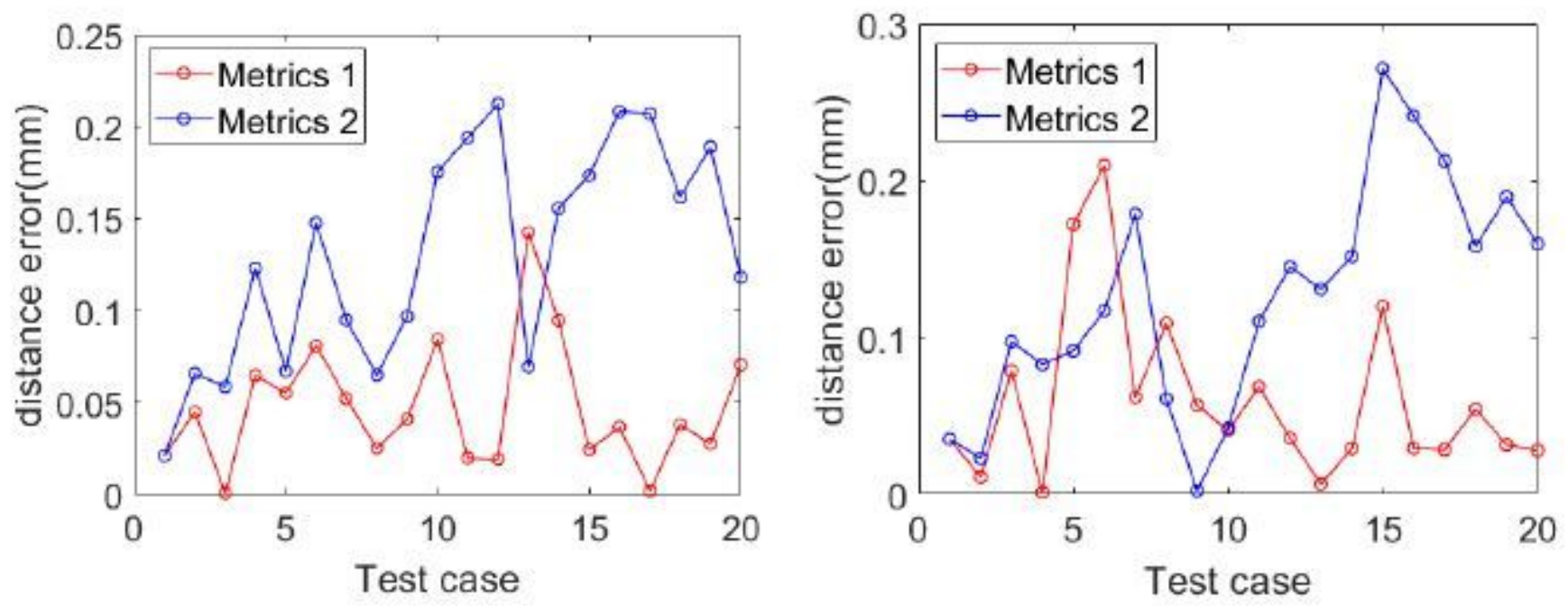

(a) Translation along $\mathrm{X}$-axis
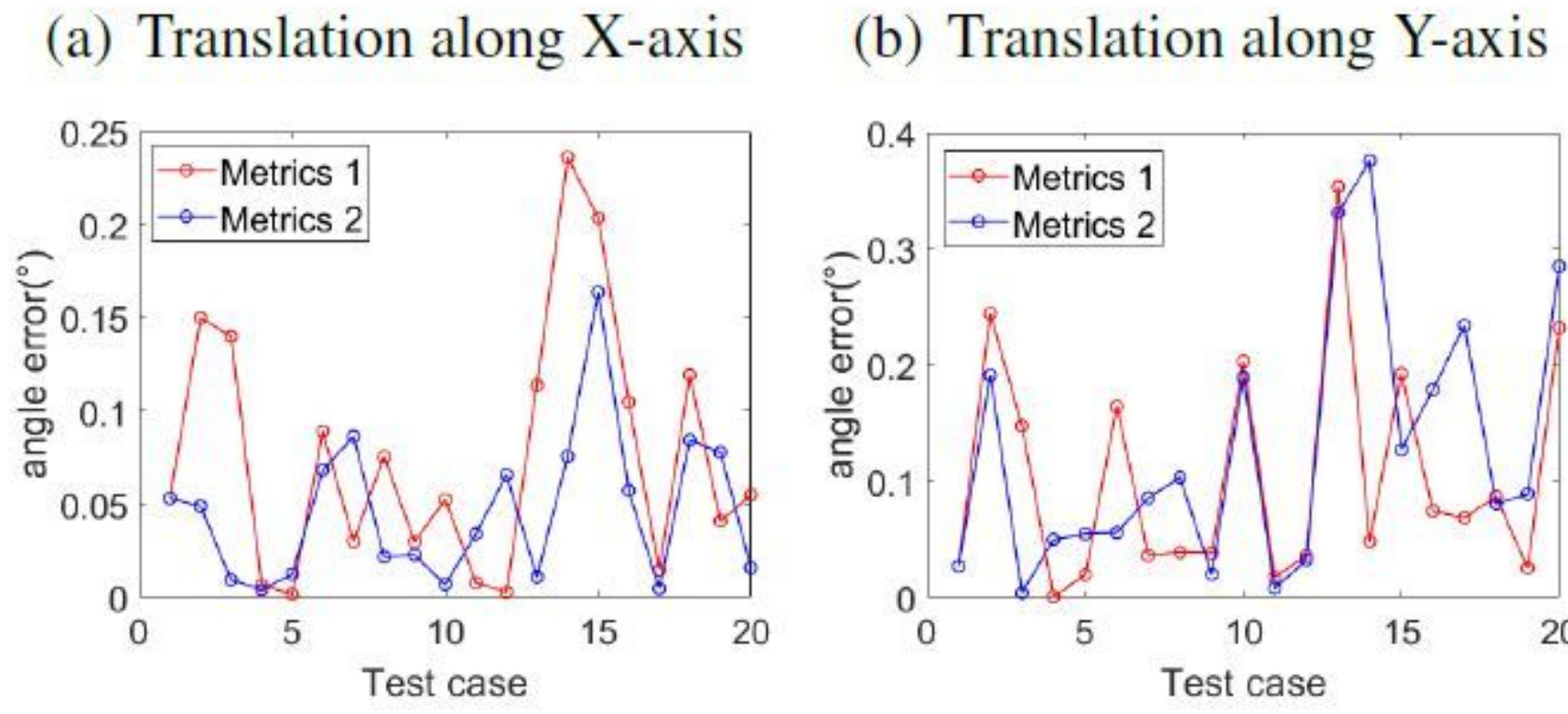

(c) Rotation around $\mathrm{X}$-axis

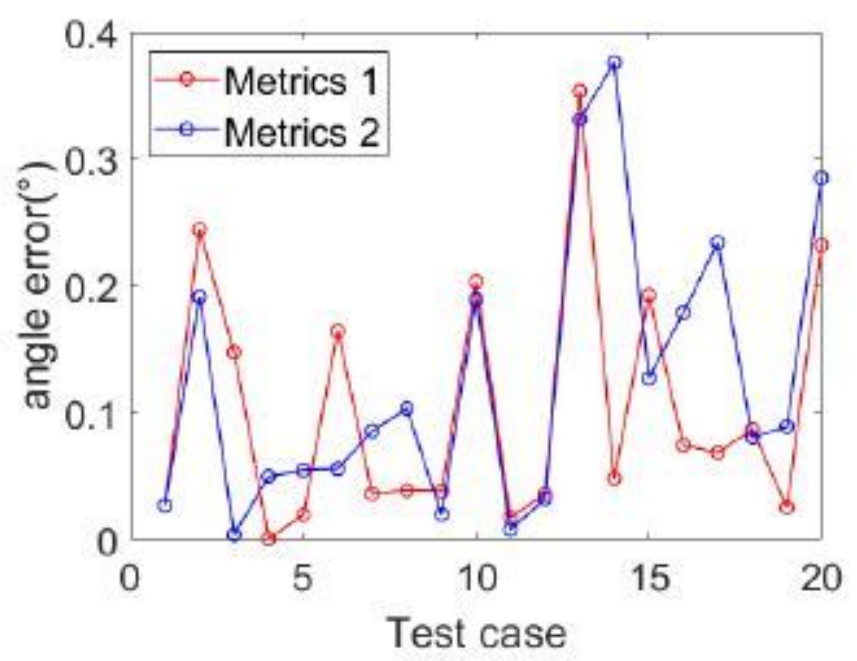

(d) Rotation around Y-axis

Figure 17

Accuracy curves for part B . 

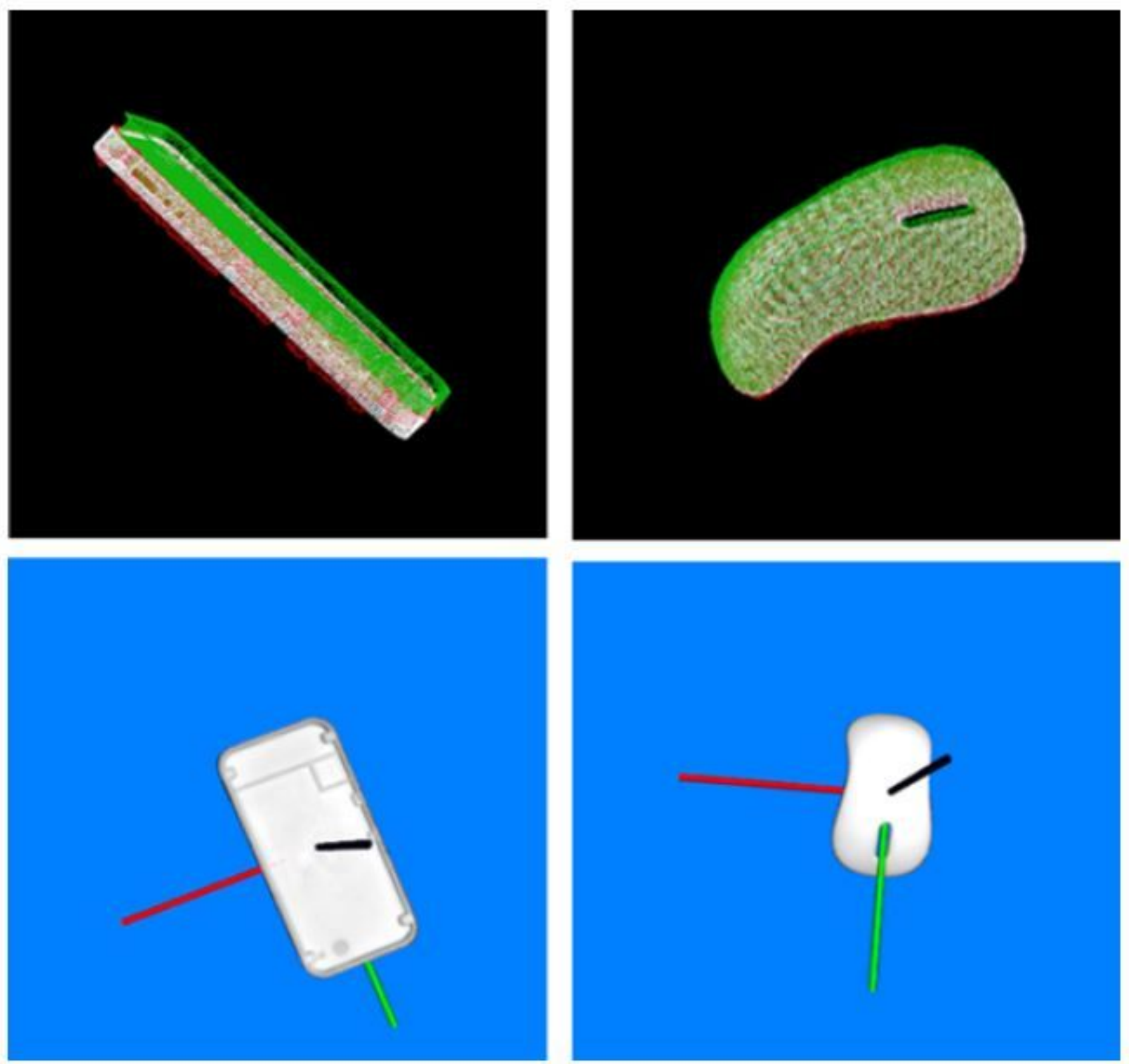

Figure 18

Pose estimation results of part A and B in the experiments: The white PCD are the complete model point clouds, the green point clouds represent the adjusted initial poses, and the red point clouds are the point clouds after registration. The bottom two pictures show the actual calculated poses 